\section{Construir una comunidad en la cárcel: cuando el conflicto motoriza y la fiesta consagra}

\author{
Gretel Schneider \\ gretel.schneider@uner.edu.ar \\ (iD) orcid.org/0000-0003-3522-2079 \\ Lucrecia Pérez Campos \\ Iucrecia.perezcampos@uner.edu.ar \\ (iD) orcid.org/0000-0002-0109-9669
}

RECEPCIÓN: 01/04/20

ACEPTACIÓN FINAL: 04/06/20

\section{Resumen}

A partir del desarrollo de proyectos de extensión en las cárceles de Paraná (Entre Ríos) de forma sostenida durante 15 años, nos proponemos analizar con un enfoque etnográfico los rasgos que cobran los lazos comunitarios y las posibilidades de construcción de una comunidad en el marco de un taller de comunicación desde la perspectiva de la comunicación comunitaria, en una unidad penal de varones. Tres escenas que mostraremos mediante registros etnográficos de los procesos nos permitirán problematizar las formas en que lo común aparece, "está siendo", se esfuma y se reinventa en la crudeza de la convivencia forzada, el encierro y la ausencia de afectos que significa estar preso. En estas condiciones nos preguntamos: ¿cuándo comienza a ponerse en juego lo común? ¿Qué estrategias nos damos como docentes y estudiantes desde la universidad para facilitar propuestas de trabajo en colaboración y que partan de un hacer a la par? ¿En qué momento comienza a realizarse lo comunitario y qué ocurre cuando esa comúnunión se ve amenazada o puesta en peligro? Estas reflexiones son parte de los resultados de un proyecto de investigación en el que, desde el Área de Comunicación Comunitaria de la Facultad de Ciencias de la Educación de la Universidad Nacional de Entre Ríos, indagamos en los sentidos que se generan en las prácticas de comunicación comunitaria que impulsamos desde la universidad.

Palabras clave: comunidad; cárcel; comunicación comunitaria; registros etnográficos; extensión universitiaria
Irene Roquel

irene.roquel@uner.edu.ar

(D) orcid.org/0000-0003-1599-4864

Universidad Nacional de Entre Ríos, Argentina.
Building community ties in prison: when conflict drives and the party enshrines

\section{Abstract}

Starting from developing extension projects sustained way for 15 years, we propose to analyze, from the ethnographic approach, the features that community ties acquire and the possibilities of building a community within the framework of a communication workshop from the perspective of Community Communication, in a male penal unit.

Three scenes that we will show through ethnographic records of the processes will allow us to problematize the ways in which the common appears, "is being", vanishes and reinvents itself in the harshness of forced coexistence, confinement and the absence of affection that means being imprisoned. Under these conditions we ask ourselves: When does the common begin to come into play? What strategies do we give ourselves as university students to facilitate collaborative work proposals and that start from making together? At what point does the community begin to take place and what happens when that common-union is threatened or endangered?

These reflections are part of the results of a research project in which, from Área de Comunicación Comunitaria of Facultad de Ciencias de la Educación, we inquired into the meanings generated in the community communication practices that we promote from University.

Keywords: community; jail; community communication; ethnographic records; university extension in the prisons of Paraná (Entre Ríos) in a
Sujetos y relaciones en extensión universitaria / Intervenciones
Construir uma comunidade na penitenciária: quando o conflito impulsiona e a festa consagra

\section{Resumo}

Partindo do desenvolvimento de projetos de extensão nas penitenciárias do Paraná (Entre Ríos) de forma sustentada por 15 anos, propomos analisar, a partir da abordagem etnográfica, as características que os laços comunitários adquirem e as possibilidades de construção de uma comunidade no âmbito de uma oficina de comunicação na perspectiva da comunicação comunitária, em uma unidade prisional masculina.

Três cenas que mostraremos através de registros etnográficos dos processos nos permitirão problematizar as maneiras pelas quais o comum aparece, "está sendo", desaparece e se reinventa na dureza da coexistência forçada, do confinamento e da ausência de afeto que significa estar preso. Sob essas condições, nos perguntamos: quando o comum começa a entrar em cena? Que estratégias nos damos como estudantes universitários para facilitar propostas de trabalho colaborativo e que comecem a ser elaboradas em conjunto? Em que momento começa a concretizar-se o comunitário, e o que acontece quando essa união comum é ameaçada ou se vê em perigo? Essas reflexões fazem parte dos resultados de um projeto de pesquisa em que, da Área de Comunicação Comunitária da Faculdade de Ciências da Educação, pesquisamos os significados gerados nas práticas de comunicação comunitária que promovemos a partir da Universidade.

Palavras-chave: comunidade; penitenciária; comunicação comunitária; registros etnográficos, extensão universitária

Para citación de este artículo: Schneider, G.; Pérez Campos, L. y Roquel, I. (2020). Construir una comunidad en la cárcel: cuando el conflicto motoriza y la fiesta consagra. +E: Revista de Extensión Universitaria, 10(12.Ene-Jun), 1-18. doi: 10.14409/extension.2020.12.Ene-Jun.9081. 


\section{Comunicación comunitaria y "ser un grupo de verdad"}

Desde el año 2005 formamos parte de un equipo que facilita talleres de comunicación y radio en la Unidad Penal $N^{\circ} 1$ Juan José O’Connor de varones de Paraná. Se trata de la cárcel más grande de la provincia de Entre Ríos; en el año 2019 su población rondaba en 1500 detenidos, en condiciones de hacinamiento, como en la mayoría de las cárceles de nuestro país (CELS, 2019). Se encuentra ubicada en el casco urbano de la ciudad de Paraná y se caracteriza por un régimen basado en procesos de elaboración de productos de consumo y servicios. Si bien es una cárcel provincial, contiene tres pabellones para presos federales que el Estado nacional financia. Comenzamos a partir de la demanda de un grupo de detenidos que conocimos en actividades culturales: querían hacer radio y "ser un grupo de verdad", expresión que nos sonó extraña y redundante.

Desde ese momento, docentes y estudiantes de Comunicación Social y Ciencias de la Educación de la Universidad Nacional de Entre Ríos (FCEdu-UNER) ingresamos semanalmente al penal para llevar adelante los espacios participativos que cobraron la denominación de "La Hora Libre" y, sus participantes, fueran presos o de la UNER, "palabreros" y "palabreras".

Al intento de garantizar el ejercicio del derecho a la educación —-preocupación que ha asumido la mayoría de las universidades argentinas (Acín y Bixio, 2016) y que constituye la condición de posibilidad de estas prácticas-, sumamos la perspectiva de la comunicación comunitaria, que es la que nos convoca. Como otros equipos universitarios, elegimos la extensión para intervenir en la cárcel (Bustelo, 2016; Delfino y Parchuc 2017; Chiponi y Manchado 2018; ACC, 2009, entre otros) puesto que consideramos que nos abre a la continuidad de procesos, al intercambio de saberes y a la posibilidad de producir en común.

La práctica tuvo cada año diferentes caminos construidos de manera participativa y dialógica, sobre todo con relación a las actividades, las formas y soportes comunicacionales que elegimos sostener. A su vez, cada ciclo que se inicia nos preguntamos acerca de los vínculos que se establecen en "La Hora Libre" entre personas que comparten la situación de cárcel y también en interacción con el equipo universitario. Considerando que los grupos fueron creciendo en magnitud hasta llegar a contar con más de 40 integrantes en el espacio de taller en los últimos años, y que los participantes no son los mismos año a año, sino que transitan con diferente grado de permanencia y compromiso, nos preguntamos: ¿es posible hablar de comunidad en el marco de la convivencia forzada del contexto carcelario? O, más bien, ¿qué tipo de comunidad se constituye en estas condiciones? O, ¿dónde aparece el sentimiento de ser una comunidad en tanto se es parte de prácticas de comunicación que se pretende comunitaria? Estas son algunas de las preguntas que hicimos respecto de los cuadernos de campo, la suma de los registros etnográficos realizados a partir de los encuentros, y desde los que pretendíamos conocer los sentidos que se generan sobre la comunicación comunitaria y los espacios que facilitamos desde la universidad en un proyecto de investigación que finalizamos en 2017, “¿De qué hablamos cuando hablamos de comunicación comunitaria?, dirigido por Patricia Fasano.

Cuando nos remitimos a la noción de comunidad, partimos de los distintos sentidos que ofrece y que nos sirvieron para constituir el Área de Comunicación Comunitaria (ACC):

"La 'comunidad' es un tipo de organización social que define y es definida a su vez, por un tipo de relación entre los miembros que la componen: 'COMÚN-UNIDAD'; de pertenencia, de identificación, de solidaridad, de hermandad, de regulación de las conductas de 
sus miembros, de vigilancia pero también de paternidad/maternidad. De allí que rescatar y promover un tipo de prácticas (comunicacionales, educativas) comunitarias signifique la opción política de apuntalar y acompañar procesos de construcción de identidad singulares, autónomos y, por ello mismo, emancipatorios". (2004:2)

Como dijimos, los registros etnográficos efectuados durante los procesos grupales nos permitirán mostrar tres situaciones para pensar lo que acontece especialmente en los mismos, lo cual juega un papel fundamental en nuestras prácticas pero adquiere distintas formas y matices: el lugar de lo común.

Creemos que lo común se encuentra en aquello que se pone en juego entre quienes venimos de afuera y quienes viven en situación de cárcel al momento del taller, y nos ponemos a hacer en conjunto y somos capaces de sostener proyectos y espacios compartidos de diálogo y negociación cultural (Freire, 2005) que superan —al menos circunstancialmentelas condiciones que nos atraviesan, por un lado, el ser universitarios, y por otro, la sujeción criminal (Misse, 2017).

Como reconocemos a la etnografía como teoría de los mundos vividos (Peirano, 2006 y 2018; Quirós, 2014 y 2018) y como enfoque que ha posibilitado que la cárcel cobre relevancia en los debates académicos en cuanto al punto de vista de quienes la habitan y/o la sufren (Kalinsky 2000; Míguez, 2008; Cerbini, 2011; Ojeda, 2008 y 2013; Encalada Falconí 2016; Ferreccio 2017), mostraremos los procesos a través de fragmentos de los diarios de campo de integrantes del equipo y problematizaremos tres escenas en las que podemos advertir las formas que asumió la comunidad en la realización de una radio abierta cada año, cuando el taller se transformó en una cátedra con un estudiante universitario preso que buscaba diferenciarse del resto y cuando un robo al interior del grupo faltó a los acuerdos y al intercambio de la confianza.

\section{La Radio Chamuyo, escenario de la comunidad}

La radio y sus posibilidades expresivas han sido, a lo largo de todos los años de trabajo, el "caballito de batalla" e hilo conductor de las diversas prácticas, la tarea y desafíos compartidos. Por esto, cada año fue coronado por una Radio Abierta Chamuyo FM, instancia semipública de muestra y fiesta final. La elección del lenguaje radial — propuesto por los primeros participantes de "La Hora Libre" — no ha sido casual: la cercanía del medio, la economía de recursos técnicos, sumadas a las características festivas y teatrales de una radio abierta, han contribuido a esta elección compartida por los sucesivos grupos.

Aquella tarde de diciembre era calurosa y el sol de la siesta partía la tierra. Por los árboles corría una leve brisa y la cancha de fútbol de la Unidad Penal № 1 se veía como un desierto de tierra y polvo. Sin embargo, alrededor de la capilla — presidida con la imagen de la Virgenhabía una actividad inusitada que transformaba el habitual silencio y quietud que ocurre cuando nadie sale de entre las rejas. Una hora antes, los palabreros comenzamos a desenrollar cables larguísimos que llegaban hasta la cocina, donde se preparó la olla de jugo dulce con hielo que se serviría más tarde con un cucharón y en vasos de plástico a todos los presentes.

Desde la facultad llegamos más temprano y con unos nervios que atravesaban nuestros cuerpos, pero la energía estaba puesta en lo que vendría. La organización de tareas era la 
misma de todos los años: traer las sillas y las mesas, colgar el cartel y reunirnos con el resto de los hacedores de esa radio que en una hora empezaría a sonar, despertando las modorras y transformando el abatimiento de los ánimos por estar presos otro diciembre más. Y otro diciembre más significa un año que termina sin la Navidad con la familia y, a su vez, una feria judicial que se avecina en enero, un mes largo en el que descansan no solo los jueces sino los pedidos de autorizaciones de salidas y de otros beneficios para la progresión del cumplimiento de las penas.

“-¡Buenas tardes! ¡Bienvenidos a la radio más buena, divertida y emocionante..! Por supuesto, ¡la Radio Chamuyo!”.

Estas son las palabras gentiles de T., quien había dejado el pabellón un rato antes y ahora, apenas abierto el micrófono, inauguraba la fiesta. Esas palabras nos ubicaron en el lugar preciso en un día excepcional; estaba comenzando la Radio Abierta Chamuyo FM en el patio de la cárcel.

El espacio al aire libre se fue llenando de público: muchas mujeres — madres, hermanas, novias, hijas - y varios niños. Trajeron tortas y mate para merendar, se sentaron en las sillas y bancos dispuestos sobre el césped a varios metros del "escenario": un tablón con sillas y micrófonos, a la manera de una cabina de transmisión de radio. También llegaron otros, ajenos al entorno: docentes, estudiantes, cronistas, y hasta las cámaras de la TV local.

Por algunas horas se multiplicaron los hombres de la guardia, ya que muchos detenidos estarían -juntos y "mezclados"- durante casi tres horas en el patio. Esto no era habitual y era "potencialmente peligroso". Sin embargo, la actitud de los penitenciarios fue rigurosamente correcta con los visitantes y se mostraron dispuestos a "sacar" (de sus celdas) a todos los protagonistas del evento y a otros espectadores, también privados de su libertad.

Desde la "mesa de transmisión", los locutores fueron anticipando los contenidos de las dos horas de programación. Al costado, el operador de la radio trató de conectarse visualmente con los conductores radiales, simulando la "luz roja" de un estudio de radio cuando habilita las voces.

A partir de ese momento vimos — sí, la radio abierta nos permite estar viendo además de escuchar - a hombres que apenas sabían leer tomar la palabra frente a un micrófono. Estrujaron el guión entre sus manos como si fuera una tabla de salvación que no los dejaría hundirse, porque durante meses habían estado trabajando en ello y porque también lo habían estudiado. Porque les pertenecía y era el momento de demostrarlo, de compartirlo y de, quizás por primera vez, hacer sonar sus cuerdas vocales en público.

Entrevistaron a los invitados y luego llegó la hora de anunciar las "noticias locas":

"Se alteró la noticia, ¡está loca! ¡El Chupacabras fue trasladado a la UP1!: después de haber realizado un raid de más de diez mutilaciones en animales domésticos, fue atrapado y alojado en la Unidad Penal Nº1 de Paraná. Lo encontraron en el bar de Pocho bebiendo caña con ruda. Más que el Chupacabras... ¡era el Chupacaña! ¡Ampliaremos!”

Algunos se sentaron junto a sus familiares y aprovecharon esta instancia como una visita extra. La "visita" es una de las dimensiones más importantes de la vida en el encierro, dado que el aislamiento es uno de sus padecimientos. 
Vimos a los participantes queriendo contar a sus allegados cómo resultaron a ser parte de la FM Chamuyo. ¿Y por qué "Chamuyo"? Este nombre fue propuesto en 2006, antes de la realización de la primera radio abierta, por uno de los participantes del grupo original. Como con todas las cosas, hicimos una votación para elegir el nombre de lo que sería la fiesta anual del taller desde entonces. Chamuyo es una palabra propia del lunfardo y que alude a esa "virtud" tan argentina de convencer con palabras, de crear realidades y no quedarse nunca sin argumentos. Entre los palabreros, decimos que sabemos distinguir: hay "chamuyo del bueno" - saber expresarse, decir lo que se siente- y "chamuyo del malo" —mentir, engatusar-. Obviamente, nuestra principal fiesta, la "vidriera" del trabajo colectivo, es siempre "chamuyo del bueno".

¿Cómo llegamos a la emisión de la Chamuyo? La fiesta chamuyera es la culminación de un proceso que requiere de aproximadamente siete meses de encuentros semanales de dos horas, del trabajo de diez facilitadores - estudiantes y docentes-y, sobre todo, de la fuerza de alrededor de 30 hombres privados de su libertad que han decidido "buscar la calle", "ser otros" o "estar en otro lugar" jugando a hacer radio.

El día de la radio, la celebración nos desafía a ocupar otros roles, a estar expuestos, a mostrarnos; de alguna manera, a ser "juzgados" ya no en un proceso judicial —en el caso de las personas privadas de su libertad- sino por la calidad de nuestro trabajo. Ese esfuerzo merece que esa tarde reluzcan las viseras nuevas, las remeras de la Radio Chamuyo de estreno, que las zapatillas se vean limpias y la mesa de la radio huela a colonia. Estar ahí es importante.

Los aplausos y los reconocimientos afirman todo esto: en ese momento se instala una visibilidad o notoriedad que antes no existía; a nuestros eventos acude la prensa y esto constituye una modesta (pero valiosa) irrupción en la agenda mediática local.

Cuando se produjo el último saludo a la audiencia, los integrantes del grupo de música tropical invitado desplegaron sus equipos, como si ese escenario fuese el del club donde actuaría el fin de semana.

Comenzó la actuación, dos de los palabreros tomaron la iniciativa e invitaron a dos de "las chicas" a bailar. Otros eran un poco reticentes, pero vencieron la timidez y se sumaron enseguida al baile. Bailar en la pista, al fin y al cabo, es una manera de "salir" y de demostrar que es nuestra fiesta. Las mujeres presentes - esposas, novias, hermanas de los palabreros- no se animaron hasta que, ante nuestra insistencia, todo se convirtió en una pequeña multitud moviéndose y gozando del final al aire libre y a toda orquesta. Los cuerpos ya no eran identificables, nos mezclamos, cambiamos de pareja, hicimos rondas y hasta un trencito que desafí las miradas del cordón de centinelas que se ocupaba de cercar la "cancha palabrera".

Cuando el sol se fue escondiendo, y después de arrebatar unos minutos más a los coordinadores y autoridades que miraban el reloj, comenzaron las felicitaciones y las despedidas. Todos nos saludamos y felicitamos; se comentaron fragmentos de la "emisión", y nos agradecieron una y otra vez. "Nos vemos el año que viene...", "que tengan una feliz Navidad junto a su familia", "que el año próximo sea bueno", o "nos vemos en la calle, porque me voy", frases en las que se destacó la fuerza del deseo ante toda traba burocrática que demorara las concreciones. "Gracias, gracias por todo, que Dios las bendiga...".

Si todavía queda alguna duda, algún resquemor, diferencia o desconfianza entre los integrantes del grupo, en este rato se diluyen y "aparece" la comunidad palabrera. Justo cuando 
se saca la foto final del grupo, la última que capturará algo de la energía y la emoción desplegada durante el año.

Todo esto también se comparte con los compañeros que no fueron protagonistas de la Chamuyo; la alegría se contagia. Aquella noche —nos dijeron otro día—, nadie durmió en el pabellón.

Después de una Radio Abierta, dijo C.:

"Sí, esa noche fue una noche que estuvimos constantemente comentando, todos prendidos en la tele con J., Cq. y G., queríamos ver cómo habíamos salido en la tele (risas); pero bueno, fue algo fantástico, la energía adentro del pabellón cambió, ese día hubo un clima totalmente distinto a los otros. Y muy contento por el recibimiento de los que se quedaron en el pabellón, tenían la misma alegría con la que volvimos nosotros".

Otro palabrero, aludiendo a esa energía contagiosa y al respeto con que fueron recibidos, aportó:

"Es algo fantástico volver al pabellón con una carga, o sea, lo que es estar encerrado no es nada fácil, pero si salís volvés con una energía, una diversión, o sea, la convivencia adentro del pabellón (...). El año pasado salieron menos internos, pero este año salimos bastantes y una energía total, el recibimiento de los que no pudieron salir también... Nos dieron un abrazo cuando entramos al pabellón, fue tremendo, una alegría entre todos, grupal... o sea, y ahí están los valores personales, como humano y como persona también. Nada más".

Al finalizar cada año, la Radio Abierta Chamuyo FM nos consagra como "grupo" —ya definitivamente "palabrero"- y nos preguntamos: ¿aquí aparece la comunidad? ¿Cuánto dura la comunidad? ¿Es solo un momento del taller o trasciende el espacio de "La Hora Libre"? ¿Podemos hablar ahora de comunidad? ¿Podemos nombrar de ese modo a este grupo que pareciera carecer de intereses comunes o de rasgos identitarios compartidos más allá de su condición de sujetos privados de su libertad? ¿Podemos afirmar que cuando culmina un "proceso en común" de alguna manera incide en su futuro?

Hemos observado, año a año, que el grupo de la Chamuyo se consolida cuando nos mostramos a otros (al afuera) en la fiesta de la Radio Abierta. No es el único momento, pero con seguridad es en este y en otras instancias festivas (y felices) cuando nos sentimos parte de un "nosotros". Desde nuestra percepción, aquí se cristaliza una comunidad que fue construyéndose poco a poco, que no es previa ni permanente, sino "precaria y provisoria".

Es decir, nos convoca un proyecto común (una referencia ya conocida de espacio universitario en la Unidad Penal), aunque su finalidad no es tan clara ni conocida por todos. Sin embargo, algo se produce en el final del proceso que reafirma "lo común".

La fiesta tiene mucho de "clausura" y de "apertura" a la vez. Cierra un proceso, el trabajo de todo un año sorteando conflictos y dificultades varias, hasta llegar a la muestra. A la vez, abre un camino que puede transitarse el próximo año, con estos $u$ otros compañeros, de la mano de otra actividad que seguramente volverá a hacer andar el mecanismo del conocimiento mutuo, los acuerdos iniciales, la obtención de la confianza.

El sentimiento de comunidad aparece —indefectiblemente- de la mano de lo gozoso, quizás porque se distingue de otras experiencias que no lo son. Tal como expresó F.:

"Yo quería decir algo del último día, de la fiesta, que siempre tiene esa dualidad que es alegría y que es tristeza también, porque yo ya sé que ahí se termina. Por lo menos 
pensaba, porque ahora me doy cuenta que podés continuar. No es necesario estar privado de la libertad para tener ataduras o estar preso. El taller de este año me sirvió para romper límites, para poder expresarme de una forma que antes yo no podía, y es como una ironía porque se da justo acá, en la cárcel. Pero me sirve para la vida, me sirve para las conversaciones que tengo con mi señora, con mis hijos, con mi mamá, con el resto de mis amistades. Mientras más uno acepta y participa, más crece, se siente mejor. Yo me siento feliz, no de estar preso, pero me siento feliz estando, porque es una decisión que uno toma, de cómo sentirse. La verdad que agradezco mucho, agradezco mucho el trabajo de todas ustedes".

Coincidimos, en primer lugar, en que lo festivo tiene la enorme virtud de "hermanar" a los participantes, de "crear una comunidad" que inaugura un nuevo rasgo de lo común.

En el momento de cierre se unifica todo el proceso, con sus conflictos y esfuerzos incluidos, en un presente donde se cristalizan los logros colectivos. Esto implica mostrar -hacia afuera- lo que somos capaces de hacer y decir. Y mostramos lo mejor que tenemos porque los destinatarios están frente a nosotros: son las madres, las hermanas, los hijos y los compañeros de pabellón.

"Mi mamá me decía que no podía creer que algo así pudiera pasar dentro de una cárcel.

Que dentro de una cárcel pudiera haber alegría, fiesta, la participación de mucha gente.

Nunca se imaginó algo así". (M.)

El otro sentido del "cierre" es — paradójicamente — la apertura a nuevos sentidos, son las actividades de recuperación del proceso, que significan nuevas instancias grupales de aprendizajes: después de la Radio Abierta — transcurrida una semana—volvemos al espacio de taller para realizar el "cierre del grupo", donde tratamos de rescatar —en una conversación desestructurada y distendida- los sentires y las voces. Para esto hemos implementado una instancia de "devolución", de especial escucha, donde procuramos que, una vez apagados los fuegos de la fiesta, podamos reunirnos a charlar (y registrar) los sentires, percepciones y preguntas que nos quedan.

Esto implica la posibilidad de volver sobre nuestras prácticas, recuperar la palabra de nuestros compañeros y "evaluar" y compartir lo trabajado.

Entonces, entre la clausura del proceso y la apertura a nuevos sentidos que incidirán en los encuentros futuros, dejamos de preguntarnos si la nuestra es o no es una comunidad (o de calificarla como precaria y provisoria), para inclinarnos a avanzar en una noción más comprensiva: a veces se produce comunidad. A nuestro entender, los momentos en que somos comunidad —cuando se fortalecen los vínculos y se refuerza una identidad comúncoinciden con las instancias felices.

Sin embargo, como veremos en el siguiente apartado, la comunidad también aparece cuando se la pone a prueba.

\section{La figura de M., "el estudiante universitario de Comunicación" en la Unidad Penal}

En el año 2016, desde el ACC formalizamos la presentación del Proyecto "Universidad y cárcel: Bitácoras y nuevos rumbos" con el objetivo de potenciar los espacios edu-comuni- 
cacionales de la FCEdu que veníamos sosteniendo desde 2005 en las cárceles, a la vez que sistematizar, profundizar y difundir el conocimiento producido con relación a la intervención universitaria en los contextos de privación de la libertad desde la perspectiva de la comunicación comunitaria y la educación popular.

Para fortalecer esta decisión y abordar la magnitud del lenguaje radial, y para sentar un antecedente académico en la FCEdu, durante 2016 fue dictado el Taller de Radio por el equipo docente especialista de la carrera de Licenciatura en Comunicación Social (a cargo del Taller de Audio I).

Nos atraía sobremanera iniciar un nuevo ciclo en la Unidad Penal habiendo por fin logrado instalar en la Facultad la idea de que era necesario y posible que se ofreciera una actividad académica intramuros como antecedente para pensar la oferta de carreras de la FCEdu en las unidades penales. Contamos con que el equipo docente de la cátedra Taller de Audio I — conformado especialmente a tal fin — pudiera dictar esa misma cátedra en la Unidad Penal de varones. Esto permitiría que uno de los internos - quien se encontraba inscripto y cursando materias libres del primer año de la Carrera Licenciatura en Comunicación Social en nuestra Facultad - accediera a cursar esa materia dentro de la Unidad, dado que, según lo establecido en el plan de estudios, dicha asignatura se puede cursar solamente en carácter de alumno o alumna regular. De no haber existido esta opción que propusimos, hubiera sido imposible para M. cursarla y acreditarla, ya que al momento no tenía habilitadas salidas de estudio ni de otro tipo.

Tener la posibilidad de dictar el Taller de Audio I en ese marco constituyó ese año la primera experiencia de dictado de una asignatura regular de una de las carreras de la UNER en el contexto carcelario; y los integrantes del equipo nos sentíamos satisfechos por haber sido precursores de esto, puesto que reconocemos cierto "atraso" de nuestra institución en cuanto a las políticas académicas de otras universidades, orientadas a garantizar el derecho a la educación de este nivel en situación de encierro.

Antes de comenzar, teníamos un numeroso listado de inscriptos que nos habían pasado desde el Área Educativa de la Unidad Penal. Algunos nombres se repetían de los talleres de años anteriores, y también estaba M., "el estudiante universitario".

"Organizados en grupos, escribir en el papel afiche aquellas palabras que relacionamos con comunicación", proponía la consigna inaugural en boca de una de las integrantes del equipo del ACC a todos los participantes del primer encuentro.

Las ideas que surgían eran varias y plasmadas no solo con letras sino también con dibujos. Nuestros registros consignan: "El afiche terminó siendo un poco de todo, palabras, dibujos, iconos (...) Papiro, caritas, radio, pentagrama". Uno de los registros decía: "Habla poco, pero en el papel se deliró".

M. estaba en su grupo, callado, observando con cierto gesto de superioridad al resto, mientras debatían la consigna. Las cejas hacia arriba, las comisuras de los labios hacia abajo, miraba desde arriba a quienes, volcando sus torsos sobre el papel extendido en la mesa, dibujaban y escribían.

Los registros de observación recuperaron la frase que también recordamos que dijo M. luego de que uno de sus compañeros le extendiera el fibrón: "La comunicación es un sueño utópico: no existe". 
M. la escribió mientras la decía, no sin antes aclarar que no sabía si hacerlo -ya que intuía que no nos iba a gustar- pero que era lo que él pensaba.

Era el primer día del Taller de Comunicación y Radio —así le llamamos- y M. —el único estudiante universitario del grupo de detenidos- se despachó ante todos nosotros - docentes y estudiantes coordinadores y resto de los participantes del taller- con la tajante afirmación de que para él la comunicación no existía porque nunca se podía saber realmente lo que el otro estaba sintiendo o pensando, aunque lo dijera...

Frente al desinterés de algunos que se alejaban dando por cerrada la actividad y la insistencia de otros en saber más sobre los motivos de su afirmación, M. comentó que para eso estudiaba comunicación. ¿Para corroborarlo? nos preguntamos en ese momento, mientras intentábamos hacerle algunas preguntas para saber mejor a qué se refería, de dónde venía ese planteo.

El mayor de los participantes de ese año —un hombre de más de 60 años que había participado de una experiencia en la radio del penal de Coronda - fue el primero en responderle y en hacerle dudar de esa afirmación — que intuíamos incomodaba a ambos-con preguntas: "Pero, ¿por qué pensás eso? ¿Cómo que no existe?

J., otro compañero, también intentó hacer una devolución a M.: "Yo no quiero sentir ni saber cómo alguien siente, sino que, si le pregunto, que me lo cuente". De esta manera notamos que, cuanto menos aparecemos en la escena quienes coordinamos el taller, con un solo disparador, la comunicación sobre la comunicación —la metacomunicación—fluye entre los participantes.

Con esa afirmación sobre la inexistencia de la comunicación y su intuición de que no nos gustaría su forma de pensar, M. empezó a mostrar lo que sería su actitud sostenida durante el tiempo que duró nuestra presencia allí ese año: por momentos desafiante, por momentos complaciente con el equipo, y distante, casi siempre muy distante, de sus pares.

¿Qué nos quería acercar con esa distancia que (im)ponía? ¿Qué quería poner en duda al compartir esa afirmación —certeza suya—? ¿Estaría pidiendo que le demostrásemos lo contrario? ¿Que le propusiéramos otra forma de concebir la comunicación, otra forma de experimentarla?

¿Cuánto de desconfianza había detrás de esa provocadora afirmación hecha a un equipo conformado por comunicadores universitarios? ¿Cuánto nos interpelaba —justamente en tanto equipo de comunicadores - que intentaba promover una perspectiva de la comunicación basada en el diálogo?

¿Es lo mismo pensar que no existe que pensar a la comunicación (comunitaria) como un sueño utópico que nos permita seguir caminando hacia un horizonte?

En los diferentes y sucesivos registros de observación que hicimos ese año, M. aparecía destacado por llegar primero o estar en el salón antes que nosotros, por estar peinado prolijamente, con el cuaderno y birome debajo del brazo, sentado detrás del resto. Cuando todos estaban en sillas dispuestas en semicírculo frente al docente, escuchando e intercambiando, M. fue aludido sentado en un lugar aparte, en silencio y tomando apuntes en su cuaderno apoyado sobre una mesa, en "actitud de estudiante universitario". Su amplio léxico y su capacidad de expresión verbal también fueron destacados en los registros como recursos utilizados por M. para diferenciarse del resto. 
Todo lo que registramos sobre $M$. aparece teñido de cierto prejuicio sobre sus actitudes en tanto ¿diferentes? ¿Diferentes de las de quién o quiénes? ¿Distinto del participante "modelo" de las prácticas de comunicación comunitaria? ¿Qué había de diferente —en esta oportunidad- en nuestro modo de construir la intervención que hacía M.? ¿Qué significaba que M. se nos presentara sobresaliendo y distinguiéndose de un "resto", ajeno a un posible y deseable "nos-otros"?

Esta fue la primera experiencia de corte más "académica", y su implementación tuvo sus particularidades. Los encuentros de ese año se organizaron de una manera peculiar: desde el equipo del ACC coordinábamos la dinámica de animación de inicio, en ronda, con el objetivo de "romper el hielo", "ponernos en clima", "distendernos" y "re-conocernos".

La propuesta siempre tenía algún componente lúdico expresivo y casi siempre implicaba "mover el cuerpo". Durante ese año, luego de finalizada la dinámica inicial, la frase inaugurada por una de nuestras compañeras en uno de los primeros talleres se repetiría en distintos encuentros: "Bueno, jahora vamos a estudiar!"; tras lo cual pasábamos a sentarnos en semicírculo en los bancos antiguos en el Salón de Usos Múltiples del penal, mirando hacia el frente, a los docentes de Audio y/o a la pantalla de proyección cuando había algún material en Power point o audiovisual que complementaba el desarrollo de la clase.

De algún modo, la frase "iahora vamos a estudiar!" marcaba momentos distintos y diferenciados dentro del espacio-tiempo que compartíamos, y también actividades diferentes. Más allá de la ronda de la primera parte, el tiempo era destinado a escuchar el desarrollo de los contenidos que compartían tanto la profesora de los "teóricos" como el profesor de "prácticos". Los docentes intercalaban su exposición con interpelaciones a los estudiantes, solicitando su participación sobre lo desarrollado pero también sobre gustos, intereses y conocimientos previos vinculados a los temas que se iban dando.

Los y las integrantes del ACC nos ubicábamos intencionalmente distribuidos por el espacio, separados unos de otros entre el grupo, e interveníamos cuando así lo considerábamos necesario, siempre que fuera con vistas a motivar la participación, evitar la dispersión, reorientar la tarea propuesta por los docentes y/o colaborar en la "decodificación" o interpretación de algún término (tanto con los docentes como con los palabreros, dado nuestro conocimiento del lenguaje académico y, en mayor o menor medida, del léxico "tumbero").

Como dijimos, en 2016 fue la primera vez que estuvimos acompañando el proceso de un estudiante que, compartiendo la condición de privado de la libertad con sus compañeros de cursado del taller y accediendo a los mismos contenidos, formalmente portaba una condición especial y diferente del resto en tanto único alumno regular de una carrera universitaria.

Paralelamente, era la primera vez que como equipo de extensión acompañábamos una propuesta de cátedra. Las diferencias en los modos de ofrecer los contenidos y de ubicarse frente a los participantes de la actividad se señalan en distintos registros de observación. También dan cuenta de un tipo de atención diferencial de los docentes de Audio hacia M., fundada sobre todo en la necesidad de que pudiera cumplir con los trabajos prácticos para regularizar la asignatura. Asimismo, la atención especial de nuestro equipo para con "el resto", más preocupados por el proceso del grupo, que no siempre incluía a M.

"Por momentos nos cuesta sostener el clima de grupalidad que acostumbramos, cuando el propio M. se autoexcluye, haciendo notar de distintas maneras al equipo y al resto de los participantes,' su lugar de único 'estudiante universitario'”. 
Al leer los registros, recordamos las veces que escuchamos a M. agradecer a los "profes" de Audio - así se dirigía M. a ellos— por "venir a darme clases a mí solo", a viva voz y delante del resto del equipo y de sus compañeros.

Sobre todo en el transcurso de la mitad del cursado, cuando ya casi no había clases teóricas, M. trabajó solo con los profesores de los prácticos; el resto de los participantes, con los integrantes del equipo del ACC, planificamos y preparamos la Radio Abierta de cierre del ciclo anual. Incluiríamos algunos de los materiales sonoros trabajados con los profes, crearíamos e improvisaríamos lo que faltara.

M. no participó casi nunca de las rondas de inicio. Llegaba, saludaba y casi enseguida se recluía con los profes en un aula aparte para concentrarse y editar, algo que los propios docentes decían necesitar para que M. cumpliera los requerimientos formales: presentar los trabajos prácticos y regularizar la materia.

De alguna manera, ese año experimentamos una doble situación de diferenciación —marginación-, exclusión interna, en esta nueva propuesta pedagógica: por un lado, entre el participante del taller en condiciones de estudiante universitario con los otros participantes $y$, por otro, entre los modos de hacer del equipo extensionista y el equipo docente de Audio, con una propuesta académica de corte más bien clásico.

M. no estaba siendo parte de la "comunidad palabrera", y eso nos llevaba a debatir al respecto al cierre de los talleres, ya que era un desafío para algunos de nosotros intentar "hacerlo parte" como uno más de la propuesta que procuraba el acceso a un derecho que consideramos debería ser común a todos.

"La comunicación es tantas cosas", decía una compañera en su primer registro. Y agregamos: también es el silencio y la distancia de M., y su planteo sobre la imposibilidad de la comunicación. Es la manifestación de singularidad que nos hace revisar la idea romántica de lo común(itario) y de la existencia de una esencia que lo defina. Es el conflicto inherente a este tipo de prácticas, sobre todo en este contexto en particular.

Una escena y un personaje nos interpelan a seguir pensando cómo incluir las singularidades, las diferencias, en un nos-otros que se pretende comunitario. ¿Cómo construir desde la universidad propuestas académicas que sean inclusivas de las diferencias y que logren evitar reproducir la segregación y discriminación interna que caracteriza a estos contextos?

La experiencia de y con M. nos devuelve la pregunta respecto de si siempre es posible construir un nos-otros o, en todo caso, ¿con qué otros? ¿Con todos los otros? ¿Qué nosotros es posible construir? ¿Qué comunidad es posible en la cárcel? ¿Cuáles son sus límites? Si alguien se nombra a sí mismo por fuera, ¿eso significa que no se produjo comunidad? Acaso los espacios que contribuimos a facilitar, ¿no pueden ser habitados de distintas maneras y allí convivir quienes se muestran más empáticos que otros? Vaya si la experiencia nos habla sobre las posibilidades de la comunicación comunitaria...

Cuando la comunidad "se pone en peligro" y hay que acudir corriendo pues... ¿se cae? Corría el mes de julio de 2017 cuando nos encontramos otra tarde en una de las actividades participativas. En grupos, teníamos que contar historias sobre nuestra relación con la radio. Era el último miércoles de taller antes de las vacaciones de invierno y por dos semanas no volveríamos al Salón de Usos Múltiples. 
En cuanto salimos, recuperamos nuestros documentos y llaves. Una de nosotras - Lucrecia- miró dentro de su mochila, buscó y rebuscó y se dio cuenta de que le faltaba el celular. Desparramando las cosas por el piso, se lo comentó a sus compañeros.

Primero, trató de recordar si realmente había traído su teléfono. Después, se preguntó si lo había perdido en el colectivo. A pesar de estas dudas, todo le hacía pensar que había desaparecido en el transcurso del taller. Con mucha precaución, para no delatar a los muchachos, volvió sobre sus pasos al salón. Se cruzó con el guardia que se encargaba de buscar a los presos de los pabellones para participar de espacios educativos y esbozó ante él una excusa: "me olvidé algo, ya salgo...".

Debajo de los bancos — donde había estado su mochila y campera— no había nada. La sospecha era abrumante y fue difícil remontar la tarde. ¿Quién había roto el acuerdo de grupo abriendo la mochila y extrayendo el celular? ¿Había sido uno solo o en complicidad con otros?

Se nos hizo evidente que todavía (después de dos meses) no nos conocíamos tanto, que el grupo era excesivamente numeroso (más de 40) y que no estaba aún consolidada la confianza. Por otra parte, sabíamos de la importancia que tiene un celular en ese contexto en tanto objeto "prohibido" pero cuya tenencia es generalizada y constituye la única posibilidad de comunicación con el exterior. Por tanto, su valor de cambio es enorme y podía convertirse en valiosos insumos: zapatillas, comida, pastillas; en suma, poder.

La pérdida -todavía no podíamos decir "hurto" o "robo"— del teléfono conmovió profundamente al equipo de la facultad y nos sumó varias dudas: ¿qué hacer? ¿Cuál era la mejor forma de resolver el conflicto, entendiendo la complejidad del contexto y los vínculos generados con los muchachos? ¿Poner en aviso a las autoridades del penal? ¿Hacer como si nada hubiera pasado? Si dábamos aviso... ¿habría requisas violentas en los pabellones? ¿pPagarían "justos por pecadores", sabiendo que en estas requisas se rompe o se pierden bienes preciados para los detenidos? Tampoco estaba ajeno el temor de ser reprendidos — por parte de las autoridades del penal— por asistir al taller con objetos que tienen el ingreso prohibido.

A su vez, si comentábamos el hecho con el grupo, ¿se armaría una especie de "cacería interna" que fuera motivo de más violencias?, ya que sabíamos que habría una reacción por parte de los que ya nos conocían. Sobre todo, sentíamos que habíamos sido traicionados en el intercambio de confianza.

Tres semanas más tarde, y luego del receso de invierno, decidimos volver y enfrentar la situación con el grupo palabrero.

En la primera ronda -muy numerosa otra vez- tomamos la palabra para expresar nuestra consternación ante el hecho. Recordamos haber dicho en una ronda: "si el primer día acordamos entre todos cumplir estos acuerdos, acá no puede haber lugar para traiciones". Enseguida percibimos que esa palabra caía como una bomba. En la pared lucía nuestro afiche con los "acuerdos" a los que habíamos arribado a principio del año y muchos se expresaron —apesadumbrados— en defensa de aquellas máximas, entre las que figuraban: "Respeto y compañerismo, Escuchar y ser escuchados, Compartir, Compromiso, Confianza". Volvimos a leerlos y a tratar de remontar el momento que, aunque liberador, fue muy intenso.

Por allí se escuchó: "nosotros lo vamos a solucionar"; y expresamente pedimos que nadie hiciera nada, que no buscaríamos culpables. Ya aliviados, volvimos a nuestra actitud habitual y expresamos que renovábamos nuestros deseos y las convicciones, que empezábamos de nuevo. 
Una semana después, según el registro de Anita, una estudiante avanzada de Comunicación Social e integrante del equipo de la Facultad, contó:

"K. y G. fueron los primeros en llegar. El último era la primera vez que venía, el miércoles pasado me habló desde 'la leonera' para decirme que está anotado y quiere venir al curso pero que siempre tiene visita y se le complica, que no lo saque de la lista. Le expliqué que no ha venido ninguna vez y eso implica que se perdió todo el proceso de las clases y conocimientos que los compañeros ya tienen, que no podía seguir faltando.

Hoy vino y, junto con K. entraron, salieron, preguntaron si había alguien más, volvieron a entrar y desde adentro me llamaron.

—iAnita! Vení un segundito... ¿Son ustedes nomás hoy? ¿La señora no viene?

Yo les respondí:

— ¿Quién? ¿Lucrecia? No, hoy no viene ella, ni Gretel, ni Irene.; tienen una reunión en la Facultad.

- ¿Pero no va a venir más?

— ¡Sí! ¡Cómo no va a venir más! Seguro el miércoles que viene, no nos puede abandonar la directora, respondí.

Salí y seguí saludando a los que iban llegando y se quedaron aprovechando el sol. Me volvió a llamar K. desde adentro. Pensé que me iba a hablar de la asistencia de G. y, cuando me acerqué, me empezó a hablar.

-Señora yo no vine la clase pasada, se acuerda que yo hablé con usted. Pero me enteré, me contaron lo que pasó con el celular.

K. agregó:

-Por eso queríamos hablar con la señora, para decirle que ya sabemos quién fue y hoy se lo va a devolver.

— ¿Cómo que ya saben? —pregunté.

-Es que el miércoles, después que hablaron de eso, el viernes en la escuela alguien me dijo. Porque es de mi pabellón y no puede ser que, siendo de mi pabellón, yo me enteré dos días después en la escuela.

- ¿Y no hablamos de que no buscaran culpables?

- Sí, señora, pero yo no puedo permitir que alguien que vive conmigo haga eso. Pero ya lo hablamos y lo va a devolver. No tenía batería, pero ya consiguió y ahora tiene, funciona. - Lo va a dejar en la entrada, yo le digo que te lo den a vos. Ya lo hablamos y se resolvió. - ¿Acaso no hablamos de resolver las cosas de otra forma?, le pregunté.

- -Sí, señora, pero acá hay códigos que ustedes pueden entender; usted, señora, con los años que tiene acá adentro, ya sabe cómo es".

Este diálogo da cuenta de la asimetría, pero también de lo común de los sentidos que circulan. Según K., nosotros estábamos en condiciones de saber que hay cosas que no se hacen: por ejemplo, al maestro no se le roba. Uno puede ser ladrón (o chorro) pero no rastrero. La práctica rastrera en la cárcel es considerada el más degradante de los robos porque se está perjudicando a quien deposita confianza en uno, con quien se comparte algo que no es material. Rastrero es quien se lleva algo que no es tan imprescindible para quien se lo lleva como para quien es su legítimo dueño y, sobre todo, con esa actitud puede quebrar un vínculo para siempre con alguien. La acción rastrera más conocida es cuando se le roba algo a un vecino. 
Esta situación nos enrostró un modo de resolver los problemas que no conocíamos dentro del penal: la negociación pacífica o el "apriete tranquilo", sin necesidad de agredir o violentar al transgresor.

A su vez, K. estaba diciéndonos que ellos nos habían permitido conocer cómo se manejaban allí y, por eso, tenían una relación de confianza y respeto con nosotros. El resto se resolvía en el pabellón.

G. se acercó a Anabel al finalizar el taller: "Anita, en la puerta te están esperando, te van a dar el celular. Es un chico con rulitos...".

Sin entender demasiado, las chicas comenzaron la actividad de taller. Todo transcurrió en forma habitual, pero no dejaban de preguntarse qué dimensión tendría ese "rescate" del teléfono. La preocupación tenía que ver con el temor de que los "aprietes" hubieran sido al mejor estilo "tumbero" y que alguien hubiera salido lastimado, cosa que queríamos evitar, a toda costa.

Cuando el equipo se retiró, en la calle las esperó una escena de película:

"Salimos. Una moto aguardaba por nosotros. Un chico, efectivamente de rulitos, y una chica atrás con el casco puesto:

- ¿Usted es Lucrecia?

—No — respondí- pero es mi compañera.

-Tenemos esto para ella.

Me dio el celular y aceleró. Me quedé con el celular en la mano casi inmóvil, mientras mis pensamientos no paraban de preguntar, preguntar, preguntar tratando de entender lo que había pasado".

Este episodio fue — quizás- el más impactante en términos de pensar los vínculos que establecemos y la percepción que tienen sus integrantes con el grupo palabrero. Nos estallaron las preguntas, más allá de la alegría de, por un lado, recuperar un objeto necesario y costoso y, por el otro, corroborar que el grupo había escuchado nuestro pesar y no se permitía haber quebrado los acuerdos.

¿Cómo se resuelven los conflictos en el ámbito carcelario? ¿Qué resortes habían saltado en este caso, que desencadenaron el "operativo rescate"? ¿Qué tipo de justicia se había aplicado aquí? ¿Quiénes habían operado en consecuencia? ¿Debíamos permitir que ellos la impartieran según sus códigos? Lo cierto es que esta situación nos llevó también a la reflexión sobre el tipo de vínculos que se establecen en este espacio y el malestar que sintieron y que los llevó a no quedarse quietos: tenían —en el marco de un ethos compartido-que encontrar el celular, devolverlo y establecer una especie de nuevo acuerdo para todos, pero en el marco de una negociación donde nadie saliera lastimado, porque así también lo planteamos nosotros.

Sintieron que el Taller estaba en peligro y actuaron en consecuencia. ¿Por qué? Así lo expresó $\mathrm{C}$. en uno de los encuentros, cuando a fin de año retomamos el tema:

"Es el respeto que se tiene hacia vos, Lucrecia, porque es algo que hablamos entre todos; bueno, se trató de solucionar entre todos, o sea, todo de buena manera, se trató, se habló, se llegó y bueno, se devolvió. Esa fue una forma de demostrar el cariño que vos nos brindás, el tiempo, todos los valores...". 
Por otra parte, una de las principales máximas que perduró durante todos los años es: "lo que es del grupo queda en el grupo". Y aquí, concretamente, se puso en primer plano; ya que si nosotros hubiéramos denunciado inmediatamente el hurto podría haber habido consecuencias indeseadas para todos, no solo para el infractor. Dijo también C.:

"Esto podría haber terminado ese día si usted hablaba allá adelante, porque no sabíamos bien quién había sido hasta que empezamos a preguntar y averiguar, de pabellón a pabellón, hasta que hablamos con esa persona que hizo mal, que tenía que hacer esto y esto y pedir disculpas. Porque ustedes venían a ayudarnos a nosotros, podíamos perder el curso. Y yo lo había hablado anteriormente con vos, Lucre, que se valoraba totalmente de parte tuya que no hayas dado aviso al servicio. Se corría el riesgo; o sea, el curso se hubiera podido cortar, fue totalmente todo discreto y ese es un valor que yo te comentaba a vos (...) que era totalmente valorable. Y nosotros, por todo lo que nos has dedicado, nos has brindado a todos... o sea, es algo que lo teníamos que hacer. O sea, nos sentíamos responsables y culpables nosotros también, ¿viste?".

Pero entonces, ¿este gesto tenía que ver con que la "víctima" era la "directora" del Proyecto?

Más allá de cierto especial respeto por la "autoridad" —la maestra, la profesora que dirige a los estudiantes-, podríamos pensar también que se produjo una reacción colectiva ante la necesidad de proteger al grupo por varios aspectos:

1) El temor a una requisa generalizada y violenta.

2) El equipo universitario también podía ser reprendido por no cumplir las reglas, como la de ingresar a una cárcel con un celular.

3) Consideraron que había una ofensa al grupo, un temor a que se perdiera la confianza que demostramos y construimos todo el tiempo. Sintieron que había que reparar el daño.

4) Sintieron temor a perjudicar al curso, a que el Servicio Penitenciario decidiera cerrar la actividad o a que decidamos no seguir con la actividad luego de aquella "traición".

5) A su vez, resolvieron no exponer y cuidar al transgresor, solucionaron el problema dentro del pabellón y no "escrachándolo" delante de todos. Intuimos además que este "apriete" no fue violento (como imaginábamos en un principio), ya que en el transcurso del año (y una vez que, de distintas maneras informales, nos enteramos de su identidad) compartimos con el infractor algunos eventos, como una obra de teatro y la RadioA Chamuyo FM.

Nadie lo puede expresar mejor que K., quien ofició de "paladín de la justicia": "Nosotros también tenemos acuerdos (...), él (por quien tomó el celular de Lucrecia) puede venir y pedir disculpas y todos necesitamos una, dos, mil nuevas oportunidades".

Por otra parte, es cierto que muchas veces idealizamos o concebimos a la comunidad desde un lugar romántico. Pero es este tipo de conflictos el que hace sentido y nos lleva a reflexionar sobre lo que significa generar comunidad en nuestras prácticas, desprendiéndonos de la idealización que a veces hacemos del término pero, aun así, manteniéndola como horizonte. 


\section{Nos quedamos pensando sobre la comunidad}

En las experiencias de prácticas de comunicación comunitaria que impulsamos en la cárcel, generalmente se produce - en forma progresiva - un reconocimiento mutuo entre quienes participamos de estas prácticas, y este reconocimiento, a su vez, solo es posible a partir de que se establece un sentimiento de pertenencia con la propuesta, ya que la forma de hacer que supone la perspectiva de la comunicación comunitaria habilita a ello.

Compartimos con Torres Carrillo (2013) que en una comunidad se crea una identidad común y que esto puede lograrse mediante la diferenciación —compartida, elaborada y socializada entre sus integrantes - o por una necesidad o mejora de un objetivo en común. También recuperamos la idea del autor sobre "lo comunitario en situaciones de borde", ya que nos permite pensar que la convivencia y vida forzada en un contexto de encierro puede concebirse como una situación "límite" o de "borde", posible generadora de acciones comunitarias. Ante la magnitud de los problemas y la inaplazable necesidad de resolver las adversidades — dice Torres Carrillo—, la activación de vínculos de solidaridad y apoyo entre los afectados supera las diferencias y distancias sociales y culturales previas al acontecimiento y habilita la emergencia de lo instituyente. De esas grietas de la cárcel como institución -y su instituido con relación a lo que se puede hacer y ser allí-, de lo que se le escapa al dispositivo de control y represión también emerge lo instituyente que, en términos de comunitas (Turner, 1999), es como un momento creativo de la vida social que el poder siempre buscará controlar, institucionalizar.

En este sentido, la Radio Chamuyo cada fin de año es un ritual que aúna, que conjura el sentimiento hacia lo grupal, es una comunidad imaginada (Anderson, 1993) que carga con un deseo colectivo pero también con lo vivenciado en una historia reciente en la Unidad Penal y "está siendo" entre logros y fricciones.

Son los conflictos, las tensiones en los procesos que, al tener resolución hacia adentro, dan cuenta de los límites de esa comunidad que preserva sus bordes y respeta ese acuerdo de "lo que es del grupo queda en el grupo", cuida sus fronteras sin exponer las fisuras, sin echar por la borda el reconocimiento "externo" o los permisos logrados (por el Servicio Penitenciario) para no hundir el barco. Esa identificación que es "lo común", ser parte de la Radio Chamuyo, nos distingue: a los presos integrantes del grupo de sus compañeros de pabellón y a estudiantes y docentes de la universidad del resto de nuestros pares, ya que decidimos intervenir en la cárcel (Schneider, 2018). También es un aporte a la transformación de la experiencia de vivir o estar en este contexto, aun partiendo de que consideramos a la cárcel como una experiencia extendida hacia quienes no necesariamente la habitan sino que están atravesados por ese aire (Ferreccio, 2017).

Pertenecer, como dijimos, implica diferenciarnos. Ser de la Radio Chamuyo, entonces, "hace al sentido de la vida" (Quirós, 2006, p. 120) en la cárcel y en la universidad.

En estas páginas advertimos cómo el grupo, a su vez, es valorado o dimensionado en sí mismo cuando se lo pone en riesgo, y en esa supervivencia o reinvención es que se la instituye (Maffesoli, 2004), por la que compartimos razones, expectativas (Appadurai, 2015) que devienen de la concreción de un proyecto. Ahora bien, ¿qué significa ser del grupo, ser un grupo de verdad? ¿Qué es lo que está en juego en esa participación?

Lo que nos sostiene es la trama que, al tensar, sostenemos; lo que nos adhiere son las emociones y lo que pasa en los encuentros, en el "presenteísmo" (Maffesoli, 2004, p. 41) 
como recorrido en el que vamos avanzando y construyendo. Comprendimos, entonces, que la comunidad acontece en tanto tenga instancias en las que peligra; que lo común se pone en juego cuando se evidencian y reflexionan de forma colectiva las rispideces. Mientras vivenciamos los encuentros, desarrollamos propuestas que van surgiendo, ensayamos y hacemos la radio. En ese mientras tanto se suceden los momentos en que "estamos siendo", en que se realiza el deseo de "ser un grupo de verdad". Y nos parece comprender que lograrlo tiene mucho que ver con el sentido que damos al espacio de comunicación comunitaria.

En todo caso, lo comunitario se asume como posibilidad de reinvención de lo social, en su posibilidad emancipadora, y en este sentido lo pensamos y hacemos desde el equipo. Puesto que la cárcel disciplina hasta los gestos y los movimientos corporales, dispone e impone, en un espacio de comunicación comunitaria apostamos a promover, ofrecer, proponer en un movimiento instituyente que asumimos como actores y actrices de lo que nos sostiene.

Subyace de nuestra parte el convencimiento que encontrar lo común que tenemos entre todos, nos acerca a un nos-otros que nos potencia y nos devuelve fortalecidos frente al individualismo y la pérdida de lazos sociales que impone el sistema. Nos permite construir otros sentidos - propios - frente a la univocidad y la homogeneización que imponen los medios masivos y ciertas instituciones. Otros sentidos sobre quiénes somos, sobre quiénes son los sujetos que circunstancialmente se encuentran privados de la libertad;; otros sentidos sobre quiénes podrían ser al correrse de las identidades estancas de "ser preso" o "ser delincuente".

\section{Referencias bibliográficas}

Acín, A. y Bixio, B. (Coords.) (2016). Sentidos políticos de la universidad en la cárcel. Fragmentos teóricos y experiencias. Filosofía y Humanidades UNC.

Anderson, B. (1993). Comunidades imaginadas. Fondo de Cultura Económica.

Appadurai, A. (2015). El futuro como hecho cultural. Fondo de Cultura Económica.

Área de Comunicación Comunitaria (ACC) (2004). Fundamentación de la creación del Área de Comunicación Comunitaria. Paraná.

Área de Comunicación Comunitaria (ACC) (Comp.) (2009). Construyendo Comunidades. Reflexiones actuales sobre comunicación comunitaria. La Crujía.

Blazich, G. (2007). La educación en contextos de encierro. Revista Iberoamericana de Educación (44), 53-60. https://www.redalyc.org/pdf/800/80004405.pdf

Bustelo, C. (2016). Experiencias de formación en contextos de encierro. Tesis de Doctorado FFyL-UBA. http:// revistascientificas.filo.uba.ar/index.php/tesis/article/view/4163

CELS (2019). Derechos Humanos en la Argentina. Informe 2019. Siglo XXI Editores.

Cerbini, F. (2011). ¿Qué es la interculturalidad en la cárcel de San Pedro (La Paz, Bolivia)? Algunas reflexiones desde "dentro". Nuevo Mundo Mundos Nuevos. http://nuevomundo.revues.org/61378

Chiponi, M. S. y Manchado, M. (2018). Prácticas culturales y comunicacionales en el encierro. La cárcel y sus sentidos en disputa. Chasqui. Revista Latinoamericana de Comunicación, 231-250.

Delfino, S. y Parchuc, J. P. (2017). Narrar para re escribir: experiencias pedagógicas en contextos de encierro. En Gerbaudo, A. y Tosti, I. (Eds.) Nano-Intervenciones con la literatura y otras formas del arte (pp. 109-142). Ediciones UNL. http://www.fhuc.unl.edu.ar/media/investigacion/centros/CEDINTEL_documentos/Nano_intervenciones.pdf\#page $=109$ 
Ferreccio, V. (2017). La larga sombra de la prisión. Una etnografía de los efectos extendidos del encarcelamiento. Prometeo.

Freire, P. (2005). Pedagogía del oprimido. Siglo XXI Editores.

Kalinsky, B. (2000). Justicia, cultura y derecho penal. Ad Hoc.

Encalada Falconí, K. (2016). Rusticidad, indígenas en la cárcel y racismo legal. Antropofagia.

Maffesoli, M. (1997). Elogio de la razón sensible. Una visión intuitiva del mundo contemporáneo. Paidós.

(2004). Juventud: el tiempo de las tribus y el sentido nómada de la existencia, Jovenes Revista de Estudios sobre Juventud, 8(20), 28-41.

Misse, M. (2017). Sujeción criminal. En Renoldi, Brígida; Álvarez, Santiago y Maldonado Aranda, Salvador (Comps.). Estado, violencia y mercado: conexiones etnográficas en América Latina. Antropofagia.

Ojeda, N. (2008). Políticas tumberas. La noción de "hacer conducta" en una colonia penal del interior de Argentina. IX Congreso Argentino de Antropología Social. Facultad de Humanidades y Ciencias Sociales. Universidad Nacional de Misiones.

(2013). El oficio penitenciario: entre lógicas burocráticas y redes de relaciones personales. Cuadernos de Antropología, (10, julio-diciembre), 315-332.

Peirano, M. (2006). A teoría vivida e outos ensaios de antropologia. Jorge Zahar Editor.

(2018). A eterna juventude da antropología: etnografia e teoria vivida. En Guber, Rosana (Coord. Gral.). Trabajo de Campo en América Latina. Tomo I. Sb Editorial.

Quirós, J. (2006). Cruzando la Sarmiento: una etnografía sobre piqueteros en la trama social del sur del Gran Buenos Aires. Antropofagia.

- (2014). Etnografiar mundos vívidos. Desafíos de Trabajo de Campo, escritura y enseñanza en antropología. Publicar, XII(XVII), 47-65. http://ppct.caicyt.gov.ar/index.php/publicar/article/viewFile/4914/5552

(2018). Por una etnografía viva. Un llamado a des-intelectualizar el "punto de vista nativo". En Guber, Rosana (Coord. Gral.). Trabajo de Campo en América Latina. Tomo I. Sb Editorial.

Schneider, G. (2018). Extensión y etnografía: una travesía por la integralidad y la comprensión de prácticas de comunicación comunitaria. +E: Revista de Extensión Universitaria, 8(9), 157-166. https://doi.org/10.14409/ extension.v8i9.Jul-Dic.7853

Torres Carrillo, A. (2013). El retorno a la comunidad. El búho. https://cpalsocial.org/documentos/632.pdf Turner, V. (1999). La selva de los signos. Siglo XXI Editores. 